\title{
ENERGY STORAGE IN MICROGRIDS: CHALLENGES, APPLICATIONS AND RESEARCH NEED
}

\author{
Hamidreza Nazaripouya ${ }^{1}$, Yu-Wei Chung ${ }^{2 *}$, and Abbas Akhil ${ }^{3}$ \\ ${ }^{1}$ University of California, Riverside Winston Chung Global Energy Research Center. \\ hamidn@ucr.edu \\ ${ }^{2}$ University of California, Los Angeles Smart Grid Energy Research Center (UCLA SMERC), \\ *Corresponding author: ywchung@ucla.edu. \\ ${ }^{3}$ Renewable Energy Ventures: abbas@ $\underline{\text { revtx.com }}$
}

\begin{abstract}
This paper studies various energy storage technologies and their applications in microgrids addressing the challenges facing the microgrids implementation. In addition, some barriers to wide deployment of energy storage systems within microgrids are presented. Microgrids have already gained considerable attention as an alternate configuration in electric power systems that can operate in grid-connected mode or islanded mode. Host grid reliability, electricity rate uncertainty, electricity demand beyond installed capacity, and regulatory and market incentives are some of the drivers motivating the deployment of microgrids. Microgrids offer greater opportunities for including renewable energy sources (RES) in their generation portfolio to mitigate the energy demand reliably and affordably. However, there are still several issues such as microgrid stability, power and energy management, reliability and power quality that make microgrids implementation challenging. Nevertheless, the energy storage system is proposed as a promising solution to overcome the aforementioned challenges.
\end{abstract}

Keywords: microgrid, energy storage system, microgrid stability, power and energy management, reliability and power quality

\section{Introduction}

Microgrids have attracted significant attention and interest in their remarkable features including operation in grid-connected and islanded modes which perfectly adapts to the modern power grid. The modernization is largely driven by the widespread deployment of Renewable Energy Resources (RES) in response to the growing power demand, depletion of fossil fuels, and increasing environmental concerns. Microgrids reliably offer a promising configuration that integrates RES as Distributed Energy Resources (DER) and mitigates the growing energy demand. 
The definition of a microgrid with respect to the size, geographic area or energy demand is not universally and uniquely stated. A broader definition of a microgrid describes it as a single controllable entity consists of interconnected loads and DER within defined electrical boundaries which operates in both grid- connected and islanded modes [1].

The features of dynamic reconfiguration and flexible operation make microgrids as a reliable and resilient source for load supplying. Microgrids can supply un-interruptible power to critical loads even during contingencies including those resulting from severe weather and natural disasters [2], [3]. In addition, microgrids play an important role in providing secure and highquality power for end users. However, design, control, and operation of microgrids are still challenging due to the RES intermittency, load profiles uncertainties, and low or lack of mechanical inertia introduced by inverter-interfaced DER. A growing body of operational experience has shown that concerns associated with operation and control of microgrids include voltage and frequency stability, reliability, and power quality, which can be addressed by incorporating energy storage into the mixed generation of the microgrid [4], [5].

Energy storage systems have been proposed as a promising solution for the operational issues of microgrids including power quality, dynamic stability, reliability, and controllability especially in the presence of RES [6]. Energy storage systems act as an energy buffer to compensate renewable intermittency, mitigate load uncertainties, and improves the microgrid stability by providing virtual inertia. The presence of energy storage in the microgrid also enhances its efficiency by managing the power flow and reducing operational losses.

This paper benefiting from the accumulated experience of real-world microgrid projects, overviews the existing challenges in control and operation of microgrids. It also studies various energy storage technologies, their characteristics and related services they can offer to microgrids. The role of energy storage systems within a microgrid to improve the stability, reliability, resiliency, and power quality as well as facilitating the energy management within microgrids is also addressed. Finally, this paper discusses the barriers need to be coped in order to expand the utilization of energy storage in microgrids.

This paper is structured as follows: Section II reviews microgrid challenges. Section III presents energy storage technologies based on their capabilities. Section IV studies the application of energy storage in microgrids along with real-world case studies. Challenges and barriers are discussed in Section V. The concluding remarks are given in Section VI.

\section{Microgrid challenges}

Although microgrid is a promising solution to provide reliable and secure power and facilitates the integration of DER units intothe power systems in an economic manner, there are still some operational concerns. In this section, the challenges including system stability, power management, power quality, and system reliability are discussed.

\subsection{Microgrid Stability}

Microgrids stability characteristics are different from those of traditional grids due to the lack of inertia in the inverter-interfaced distributed generation (DG) [7], [8]. The deployment of inverter-interfaced DGs in microgrids has led to the major operating differences between a microgrid and a traditional grid in time response and inertia. These differences lead to complication in operation and stability of microgrids.

A traditional power grid stores a significant amount of kinetic energy in the rotating mass which contributes to grid stability, while inertia-less DGs do not. The kinetic energy compensates for the short timescale mismatches of demand and supply and thus maintains the stability of the grid voltage and frequency. 
Since a microgrid operates in grid-connected and islanded mode, the microgrid stability is studied in these two modes [8] and discussed separately.

In mode of grid-connected, microgrid absorbs energy from the main grid when there is a deficit in its internal energy balance and sends it back to the grid when it has a surplus generation. In this case, the main grid balances the mismatch between the generation and load and ensures the system frequency stability. Therefore, in grid-connected mode, rather than rotor angle and frequency stability, voltage stability is the main issue. To this end, small and large disturbances (transient) analysis are performed only for the voltage.

In islanded mode, since a microgrid is electrically independent of the main grid, it has the responsibility to maintain both voltage and frequency stability.

The transient stability of a microgrid at the time of islanding is investigated in [9], and the results show that the microgrid can lose stability very easily due to the power imbalance while even load shedding might not be an effective solution to stabilize the system.

\subsection{Microgrid Power Management}

A robust and autonomous power management system is of vital importance to enable the deployment of microgrids within the current grid and accelerate their adoption by utilities and customers. The role of a power management system is to control the power flow while optimizes an objective function such as fuel consumption, efficiency, and overall operation costs $[10,11]$. However, the hybrid AC/DC architecture [12] and multi- operational modes of a microgrid make the microgrid power management complicated. Firstly, AC/DC hybrid microgrids require proper coordination among the AC and DC generators. Secondly, supply and demand balancing during the transition from grid- connected to islanded mode should be effective to make the transition seamless. Finally, the incorporation of intermittent RES such as solar and wind as well as stochastic and uncertain loads such as electric vehicles (EVs) into microgrids[13] pose challenges to microgrid power management. The above- mentioned physical configuration and components of a microgrid impose several constraints for power management and thus, turn it into a complex multi-objective optimization problem[14].

\subsection{Microgrid Power Quality and Reliability}

Power quality in electric grid is defined as maintaining the magnitude and frequency of the voltage within the allowable range of rated value in a sinusoidal waveform [15]. In a microgrid comprised of a collection of DGs, providing high power quality can be challenging due to the following reasons. First, most DGs usually interconnect via power electronics. The nonlinear voltage-current characteristic of these inverter-interfaced DGs generates harmonics in the system, negatively affects the power quality. Second, high penetration of DGs in the microgrid, which are mostly RESs such as solar or wind power, can degrade the power quality significantly because of their nature of intermittency and the reverse power flow. Lastly, nonlinear and stochastic loads, loads with considerable reactive power, and transition between grid- connected and islanded modes are other factors that lead to power quality issues in microgrids. All of those may result in the issues including power variation, voltage and frequency deviation, voltage sag, voltage swell, voltage flicker, poor power factor, Total Harmonic Distortion (THD), and unbalanced voltage and current, which are power quality concerns in microgrids [16].

The initial motivation of developing and deploying microgrids is to improve power system reliability. The capability of microgrids to improve reliability relies on the availability of DERs and dynamic response of local generation units to withstand sudden disturbances or faults. However, the reliability improvement in microgrids is still challenging as the DERs are 
stochastic and non- dispatchable resources. That is, complex scenarios including multistochastic factors such as loads, supply, and failure events should be managed to ensure system reliability [17].

\section{Energy Storage Technologies}

Energy storage is a device that is capable of converting the electrical energy to a storable form and convert it back to electricity when it is needed. Based on the form of stored energy, there are four main categories for energy storage technologies: Electrical Energy Storage (EES), Mechanical Energy Storage (MES), Chemical Energy Storage (CES), and Thermal Energy Storage (TES) as depicted in Table 1.

Table 1. The Classification of Energy Storage Technologies.

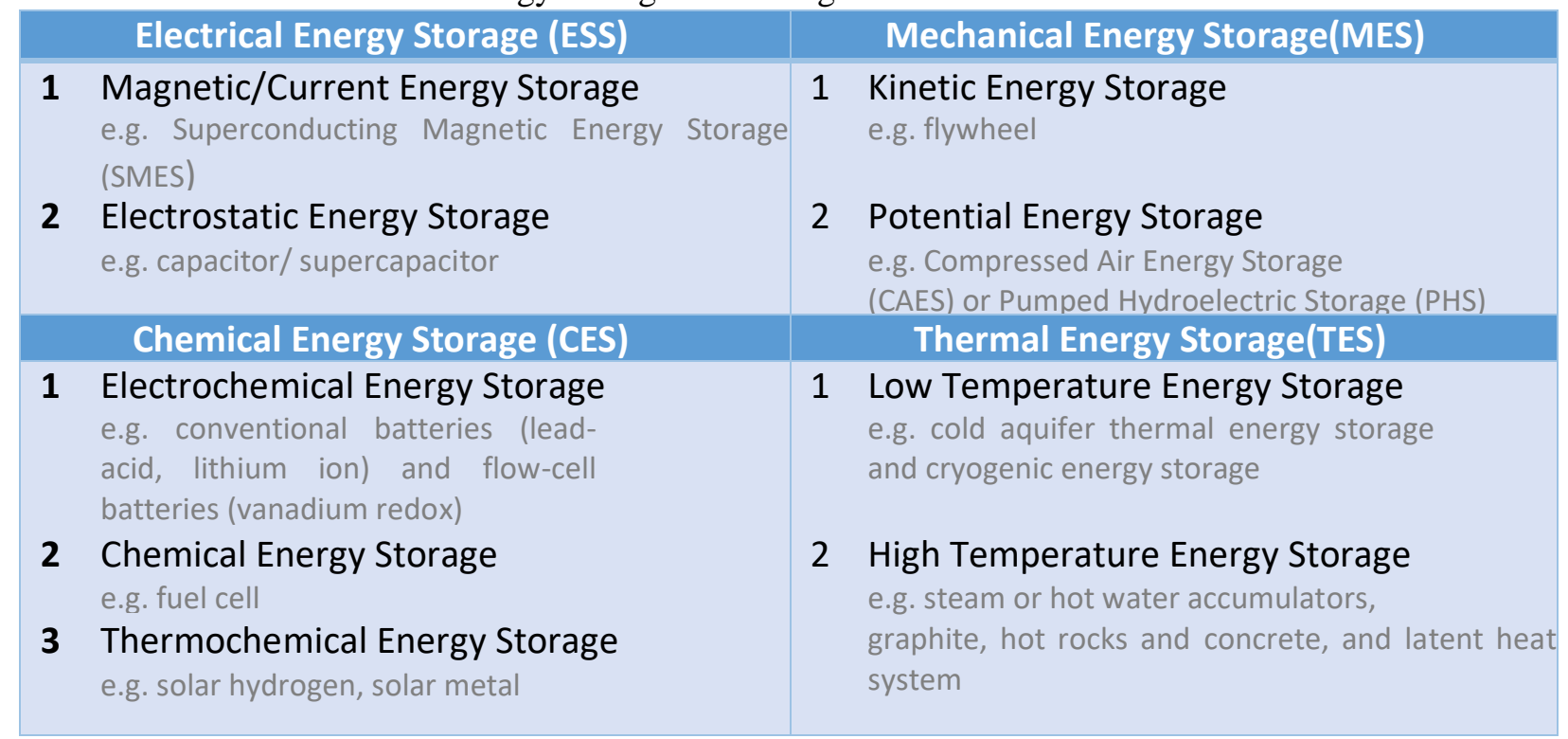

The functionalities and applications of energy storage technologies depend on their characteristic including rating characteristic, dynamics, space requirement, and performance. The rating characteristic is determined by power and energy rating. Power rating refers to the charge/discharge rate while energy rating represents the discharge duration. The dynamics can be evaluated by response time and ramp rate. The response time is the time duration that energy storage goes from zero discharge to full and the ramp rate is the rate at which the output power can change. The space requirement depends on power and energy densities, and the performance can be expressed as the efficiency of the energy storage. Table 2 shows the common energy storage technologies deployed in microgrids and their characteristics.

\section{Applications of Energy Storage in Microgrids}

The integration of intermittent RES within a microgrid has turned the traditional power generation from controllable and dispatchable recourses into uncontrollable and nondispatchable ones. In addition, stochastic EV loads make the power demand unpredictable. Therefore, microgrids encounter a big challenge for balancing the power demand and supply. Energy storage, however, becomes a capable solution for microgrids to address the mismatch of the unpredicted power demand and generation. Energy storage system also plays an indispensable role in microgrids to ensure the system stability, reliability and power quality 
while lower the operation cost and enhance the power efficiency. In this section, different capabilities and applications of energy storage that can address the challenges mentioned in section II are discussed and presented.

\subsection{Stability Enhancement}

Energy storage systems connected to power electronic device can be utilized to improve both voltage and frequency stability by exchange active and reactive power with a microgrid.

Under incidents such as reactive power shortage, dynamic variation of load and generation, and operation of load tap changers and voltage regulators, as the main sources of voltage instability, there is a need for a mechanism to maintain the voltage within an allowable range for stable operation of microgrids. It should be noted that since in a microgrid, the line resistance to line reactance ratio $(\mathrm{R} / \mathrm{X})$ unlike the transmission system is considerable, the impacts of active and reactive power on frequency and voltage are not decoupled [18]. Fortunately, the battery energy storage system as a source of active and reactive power can be controlled to compensate the imbalance of active and reactive powers with fast dynamics.

Table 2. Characteristics of various energy storage technologies utilized in microgrids.

\begin{tabular}{|c|c|c|c|c|c|c|c|}
\hline \multirow{3}{*}{$\begin{array}{c}\text { Energy } \\
\text { Storage } \\
\text { Technology }\end{array}$} & \multicolumn{7}{|c|}{ Characteristics } \\
\hline & \multicolumn{2}{|c|}{ Rating Characteristic } & \multicolumn{2}{|c|}{ Dynamics } & \multicolumn{2}{|c|}{$\begin{array}{c}\text { Space } \\
\text { Requirements }\end{array}$} & Performance \\
\hline & $\begin{array}{l}\text { Discharging/ } \\
\text { Charging rate } \\
\text { (MW) }\end{array}$ & $\begin{array}{l}\text { Discharging } \\
\text { duration }\end{array}$ & $\begin{array}{l}\text { Response } \\
\text { time }\end{array}$ & Ramp rate & $\begin{array}{l}\text { Energy } \\
\text { Density } \\
\text { (Wh/kg) }\end{array}$ & $\begin{array}{l}\text { Power } \\
\text { Density } \\
\text { (W/kg) }\end{array}$ & Efficiency (\%) \\
\hline $\begin{array}{l}\text { Battery Energy } \\
\text { Storage(BES) }\end{array}$ & $0-40$ & ms - hrs & $\mathrm{ms}$ & $\mathrm{MW} / \mathrm{sec}$ & $10-250$ & $70-300$ & $70-90$ \\
\hline $\begin{array}{l}\text { Capacitors } \\
\text { (Supercapacitors) }\end{array}$ & $\begin{array}{l}0.01-0.05 \\
(0.01-0.3)\end{array}$ & Ms $-1 \mathrm{hr}$ & $\mathrm{ms}$ & $\mathrm{MW} / \mathrm{sec}$ & $\begin{array}{l}0.05-5 \\
(0.1-15)\end{array}$ & $\begin{array}{c}100 \\
000 \\
(500- \\
10,000)\end{array}$ & $\begin{array}{c}60-90 \\
(75-95)\end{array}$ \\
\hline $\begin{array}{l}\text { Flywheel Energy } \\
\text { Storage(FES) }\end{array}$ & $0.002-0.25$ & $\mathrm{~ms}-15 \mathrm{~min}$ & Instantaneou & $\mathrm{MW} / \mathrm{min}$ & $5-130$ & $\begin{array}{l}400- \\
1500\end{array}$ & $90-95$ \\
\hline Fuel Cell & $0.001-50$ & Sec - 24+ hr & $\mathrm{ms}$ & $\mathrm{MW} / \mathrm{min}$ & $\begin{array}{l}800- \\
10,000\end{array}$ & $500+$ & $20-90$ \\
\hline $\begin{array}{l}\text { Compressed Air } \\
\text { Energy Storage } \\
\text { (CAES) }\end{array}$ & $0.1-300$ & $1-24+h r$ & $\min$ & $\mathrm{MW} / \mathrm{min}$ & $3-60$ & - & $42-89$ \\
\hline $\begin{array}{l}\text { Superconducting } \\
\text { Magnetic Energy } \\
\text { Storage (SMES) }\end{array}$ & $0.1-10$ & $\mathrm{~ms}-10 \mathrm{sec}$ & $\begin{array}{c}\text { Instantaneou } \\
\mathrm{s}\end{array}$ & $\mathrm{MW} / \mathrm{ms}$ & $0.5-5$ & $\begin{array}{l}500- \\
2000\end{array}$ & $>97$ \\
\hline $\begin{array}{l}\text { Pumped } \\
\text { Hydroelectric } \\
\text { Storage (PHS) }\end{array}$ & $0.1-5000$ & $1-24+h r$ & $\mathrm{sec}-\min$ & $\mathrm{MW} / \mathrm{sec}$ & $0.5-1.5$ & - & -85 \\
\hline
\end{tabular}

An energy storage system can also act as virtual inertia in a microgrid to enhance the frequency stability by compensation the low inertia of RES $[19,20]$. That is, the energy stored in the storage systems emulates the kinetic energy stored in the rotor of synchronous generator which can be released in the events of disturbance or drastic demand- supply imbalance. Battery energy storage systems, supercapacitors, superconducting magnetic energy storage (SMES), and flywheel Energy Storage (FES) are suitable candidates for dampening the frequency oscillations in microgrids due to their fast dynamics. 


\subsection{Energy Management}

Energy management in a microgrid is an economical strategy of dispatching DERs and managing the loads. Energy management not only minimizes the operation cost but also meets the load demand within the microgrid operational constraints. Energy storage can be viewed as a dispatchable source and controllable load in energy management systems. Since it is a challenge to balance the power supply and demand in a microgrid with its unpredictable RES generation and stochastic loads, energy storage system plays an indispensable role to provide more flexibilities for relaxing the energy management constraints and optimizing the objective function [21].

Energy storage can offer different services to facilitate microgrid energy management. Firstly, as a dispatchable and controllable prosumer, they can be used to ease the complexity of energy management problem in the presence of intermittent RES and stochastic loads by smoothing the RES power generation and managing the demand [22]. Secondly, energy storage systems are capable of shifting the time of power generation and demand. Many of RES might generate a considerable amount of energy when the demand is low, and thus result in energy generation surplus [23]. Without energy storage, the surplus energy may be curtailed. Therefore, an energy storage system can store the energy when the demand is low (extra generation) and release the energy when the demand is high (energy shortage). With this capability, energy storage systems can provide microgrids with services such as peak shaving, load leveling, and energy arbitrage. They can also prevent curtailment of renewable energy [23]. Lastly, with optimal placement of energy storage systems within a microgrid, they can help to reduce power loss and improve efficiency through maximizing the local energy utilization and reducing the transferred power from the main grid [18]. In addition, since in microgrids the energy loss in conductors and electrical equipment is proportional to current squared $\left(\mathrm{RI}^{2}\right)$, distributing loads evenly over time and reducing the peak demand by energy storage can reduce the energy loss. Finally, energy storage systems by providing reactive power locally, can also decrease the current drawn by loads from resources and reduce the loss over lines.

\subsection{Power Quality Improvement}

Load characteristics, variability in power generation, operation of switching devices, and malfunctioning of equipment within a microgrid may degrade the power quality and thus negatively affect the operation of system through reducing the efficiency and increasing the maintenance cost in microgrids. Energy storage systems can be deployed to assist power quality improvement by offering services such as renewable intermittency compensation, voltage support, power factor correction, phase balancing, and harmonic compensation, which are described below.

\subsubsection{Renewable Intermittency Compensation:}

The intermittency in power generation can be effectively mitigated with a fast response energy storage system integrated with RES [24]. An energy storage unit can automatically smooth the variation in RES by controlling the ramp-rate through instantaneous energy exchanging. Figure 1 depicts the obtained result from using energy storage to smooth solar power in Borrego Spring microgrid [25]. 


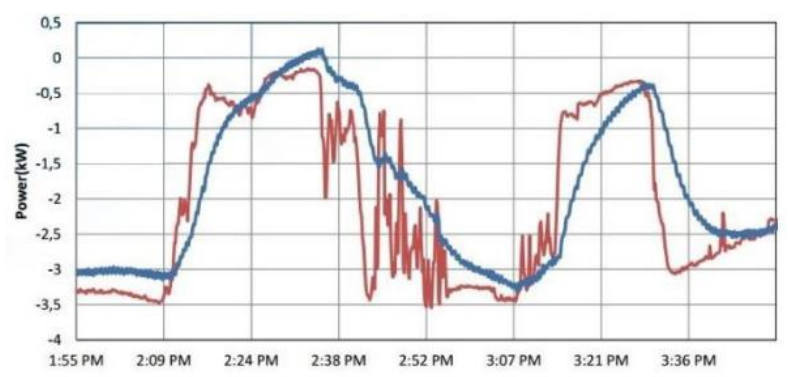

Figure 1. Smoothing the power of PV solar using energy storage in Borrego Spring microgrid [25]

\subsubsection{Voltage Support:}

Voltage sag/rise is considered as a power qualityissue in microgrids. Voltage sag occurs when the capacity of the system in delivering the required energy is not adequate to meet the demand. Voltage rise is due to reverse power flow when the local generation exceeds the consumption. Voltage variation may cause shutting down or malfunctioning of electric equipment. The inverter-interfaced energy storage systems by operating in four-quadrant (charging, discharging, leading or lagging) and controlling active and reactive power in the microgrid can effectively regulate the voltage [18].

\subsubsection{Power Factor Correction:}

Energy storage systems equipped with power electronic converters are valuable assets within a microgrid to improve power factor and compensate the effects from local reactance. In [26] distributed battery energy storage systems are optimally scheduled to mitigate marginal loss through power factor correction.

\subsubsection{Phase Balancing:}

A three-phase microgrid might contain single-phase loads and generation. Therefore, for a safe and efficient operation of the system, balancing loads and generation among phases is needed. Single-phase energy storage systems can mitigate the phase imbalance if they are separately integrated to each phase and exchange active and reactive power independently [27].

\subsubsection{Harmonic Compensation:}

The widespread deployment of intermittent RES and nonlinear loads such as fluorescent lamp, motor drivers, and electric vehicle chargers leads to current and voltage harmonics in the system. The harmonics can be compensated by proper switching of energy storage power electronic converters to act as an active filter and producing the inverse of harmonics in the system [28].

\subsection{Reliability Improvement}

Power reliability can be defined as the ability to provide sustainable power to meet the consumer's electricity demand. According to reliability indices defined by Institute of Electrical and Electronics Engineers (IEEE) [29], one of the key factors in reliability degradation is power interruption. Microgrids with the support of energy storage system is a promising solution to improve the power reliability. In the event of the outage, the energy storage system provides the consumers with the required power to ride-through the outage until the backup generation 
starts up and the system continues the normal operation [30]. The microgrid energy storage in order to prevent power interruption during the transition into the islanded mode of operation can also offer the ride-through and bridging services.

The other influential factors in microgrid reliability include capacity availability and resources adequacy. The required generation capacity for a microgrid usually is about 115 percent of its forecasted peak demand. Adding more dispatchable generation is the common practice to provide generation capacity. However, the energy storage system is a competitive alternative to provide resource adequacy.

\subsection{Resiliency Improvement}

Microgrid resiliency can be defined as the ability to endure and recover from disruptive events and to minimize the duration, intensity, and the negative impacts of disruptions [31]. Energy storage can be controlled properly to manage the network power flow and balance the supply and demand in order to stabilize the system during the contingencies [32]. To sustain the sudden changes, energy storage systems can provide virtual inertia to the microgrid, which enhances the robustness of the entire system. In addition, the integration of RES with energy storage system turns the intermittent RES generation into a reliable energy source to provide power during grid outages.

\section{Challenges and Barriers}

The barriers and challenges for widespread deployment of energy storage system in a microgrid are discussed in this section.

There are three major issues for the extensive deployment of energy storage. The first issue is the deployment cost. Due to the high cost of energy storage systems, the value stream and revenue created by energy storage systems within a microgrid should be clearly identified and quantified. It should be noted that besides the cost of energy storage technology, the cost of ancillary equipment as well as system installation, integration, and commissioning should be reduced. Sometimes, the secondary cost reaches up to 60-70 percent of the total cost [32].

Choosing the proper type, sizing and placement of energy storage within a microgrid is the second challenge. The first difficulty that users may encounter is to decide the best fit technology for their application from a wide range of selections of different energy storage systems with different characteristics. Even if they select one, the sizing of the energy storage capacity would be challenging. Therefore, to facilitate energy storage utilization, creating a comprehensive standard to be able to evaluate and compare the quality and performance of different technologies is inevitable. Next step is to develop a clear guideline to help energy storage users with the optimal selection of energy storage type and size and optimal placement in order to gain the maximum benefit. Lastly, appropriate rules and regulation should be formed to promote energy storage deployment in the microgrids and encourage stakeholders' investment. Clear market models, as well as adequate incentives should be provided by regulatory entities for investors, while the regulatory restrictions that prevent stakeholders to collect revenue need to be removed. Moreover, a clear and accurate evaluation of energy storage systems performance is required for stakeholders to assess the investment. To this end, desired performance criteria should be defined through establishing appropriate codes and standards and also a unified framework for analysis and reporting the performance of energy storage should be developed to alleviate the uncertainties over capabilities of energy storage and make the stakeholders confident to invest. 


\section{Conclusion}

This paper provides an overview of microgrid challenges including stability, power and energy management, reliability and power quality. The paper also studies and compares various energy storage technologies based on their characteristics such as rating characteristics, dynamics, space requirement, and performance. The role of energy storage systems in microgrids and services that they can offer to address the challenges are discussed. Finally, the barriers of promoting energy storage system in microgrids are discussed. Technical barriers such as determining the best technology fit, sizing and placement problems, along with non-technical barrier including high cost, lack of rules, standards, and regulation are the issues need to be overcome in order to allow widespread propagation of energy storage. There is no question that the energy storage system will be an indispensable component of future microgrids. Once conquering the barriers, new markets and applications will be opened for energy storage.

\section{References}

[1] Dan T. Ton and Merrill A. Smith, The U.S. Department of Energy's Microgrid Initiative. The Electricity Journal, 2012. 25(8): p. 84-94.

[2] Models Microgrids-Benefits, Barriers and Suggested Policy Initiatives for the Commonwealth of Massachusetts. KEMA Inc.: Burlington, MA, USA, 2014.

[3] Nikos Hatziargyriou, Hiroshi Asano, Reza Iravani, and Chris Marnay, Microgrids. IEEE power and energy magazine, 2007. 5(4): p. 78-94.

[4] Sina Parhizi, Hossein Lotfi, Amin Khodaei, and Shay Bahramirad, State of the art in research on microgrids: A review. IEEE Access, 2015. 3: p. 890-925.

[5] Mariya Soshinskaya, Wina HJ Crijns-Graus, Josep M Guerrero, and Juan C Vasquez, Microgrids: Experiences, barriers and success factors. Renewable and Sustainable Energy Reviews, 2014. 40: p. 659-672.

[6] Qiang Fu, et al., The Role of Energy Storage in a Microgrid Concept: Examining the opportunities and promise of microgrids. IEEE Electrification Magazine, 2013. 1(2): p. 21-29.

[7] Ramon Zamora and Anurag K Srivastava, Controls for microgrids with storage: Review, challenges, and research needs. Renewable and Sustainable Energy Reviews, 2010. 14(7): p. 2009-2018.

[8] Zhikang Shuai, et al., Microgrid stability: Classification and a review. Renewable and Sustainable Energy Reviews, 2016. 58: p. 167-179.

[9] Ritwik Majumder, Some aspects of stability in microgrids. IEEE Transactions on power systems, 2013. 28(3): p. 3243-3252.

[10] Yubo Wang, Bin Wang, Chi-Cheng Chu, Hemanshu Pota, and Rajit Gadh, Energy management for a commercial building microgrid with stationary and mobile battery storage. Energy and Buildings, 2016. 116: p. 141-150.

[11] Yubo Wang, Wenbo Shi, Bin Wang, Chi-Cheng Chu, and Rajit Gadh, Optimal operation of stationary and mobile batteries in distribution grids. Applied Energy, 2017. 190: p. 1289-1301. 
[12] Farzam Nejabatkhah and Yun Wei Li, Overview of power management strategies of hybrid AC/DC microgrid. IEEE Transactions on Power Electronics, 2015. 30(12): p. 7072-7089.

[13] Bin Wang, et al., Predictive Scheduling Framework for Electric Vehicles With Uncertainties of User Behaviors. IEEE Internet of Things Journal, 2017. 4(1): p. 52-63.

[14] CM Colson and MH Nehrir. A review of challenges to real-time power management of microgrids. in Power \& Energy Society General Meeting, 2009. PES'09. IEEE. 2009. IEEE.

[15] Ewald Fuchs and Mohammad AS Masoum, Power quality in power systems and electrical machines. 2011: Academic press.

[16] Arangarajan Vinayagam, KSV Swarna, Sui Yang Khoo, and Alex Stojcevski, Power Quality Analysis in Microgrid: An Experimental Approach. Journal of Power and Energy Engineering, 2016. 4(04): p. 17.

[17] Scott Kennedy and Mirjana Milosevic Marden. Reliability of islanded microgrids with stochastic generation and prioritized load. in PowerTech, 2009 IEEE Bucharest. 2009. IEEE.

[18] H Nazaripouya, Y Wang, P Chu, HR Pota, and R Gadh. Optimal sizing and placement of battery energy storage in distribution system based on solar size for voltage regulation. in Power \& Energy Society General Meeting, 2015 IEEE. 2015. IEEE.

[19] Sumiti Lamichhane, H Nazaripouya, and S Mehraeen. Micro grid stability improvements by employing storage. in Green Technologies Conference, 2013 IEEE. 2013. IEEE.

[20] Toshinobu Shintai, Yushi Miura, and Toshifumi Ise, Oscillation damping of a distributed generator using a virtual synchronous generator. IEEE transactions on power delivery, 2014. 29(2): p. 668-676.

[21] Yubo Wang, et al. Optimal energy management for Microgrid with stationary and mobile storages. in Transmission and Distribution Conference and Exposition (T\&D), 2016 IEEE/PES. 2016. IEEE.

[22] Hasan Geramifar, Majid Shahabi, and Taghi Barforoshi, Coordination of energy storage systems and DR resources for optimal scheduling of microgrids under uncertainties. IET Renewable Power Generation, 2016. 11(2): p. 378-388.

[23] Alireza Majzoobi and Amin Khodaei, Application of Microgrids in Supporting Distribution Grid Flexibility. IEEE Transactions on Power Systems, 2016.

[24] MJE Alam, KM Muttaqi, and D Sutanto, A novel approach for ramp-rate control of solar PV using energy storage to mitigate output fluctuations caused by cloud passing. IEEE Transactions on Energy Conversion, 2014. 29(2): p. 507-518.

[25] Neal Bartek, Borrego Springs Microgrid Demonstration Overview. 2015: Society of American Military Engineers, San Diego, CA.

[26] Ali Moeini, Innocent Kamwa, and Martin de Montigny, Power factor-based scheduling of distributed battery energy storage units optimally allocated in bulk power systems for mitigating marginal losses. IET Generation, Transmission \& Distribution, 2016. 10(5): p. 1304-1311. 
[27] Sun Sun, Ben Liang, Min Dong, and Joshua A Taylor, Phase Balancing Using Energy Storage in Power Grids Under Uncertainty. IEEE Transactions on Power Systems, 2016. 31(5): p. 38913903.

[28] Sharad W Mohod and Mohan V Aware, A STATCOM-control scheme for grid connected wind energy system for power quality improvement. IEEE systems journal, 2010. 4(3): p. 346-352.

[29] Transmission and Distribution Committee, IEEE Guide for Electric Power Distribution Reliability Indices. IEEE Std 1366 ${ }^{\mathrm{TM}}-2003,2003$.

[30] R Arghandeh, Manisa Pipattanasomporn, and Saifur Rahman, Flywheel energy storage systems for ride-through applications in a facility microgrid. IEEE Transactions on smart grid, 2012. 3(4): p. 1955-1962.

[31] Sayonsom Chanda and Anurag K Srivastava, Defining and enabling resiliency of electric distribution systems with multiple microgrids. IEEE Transactions on Smart Grid, 2016. 7(6): p. 2859-2868.

[32] C Gouveia, J Moreira, CL Moreira, and JA Pecas Lopes, Coordinating storage and demand response for microgrid emergency operation. IEEE 